科 学 通 报

\title{
$\mathrm{ErCo}_{4}$ 原子簇的电子结构及磁性研究 ${ }^{*}$
}

\author{
李 华 姜寿亭 梅良模 高汝伟 \\ (山东大学物理系, 济南 250100)
}

\section{关键词准晶态、非晶态、正四面体、MS-X $\mathbf{X}_{\alpha}$ 方法、磁性质}

快淬合金 $\mathrm{Er}_{x} \mathrm{Co}_{100-x}(x \approx 20)$ 是一种强磁性材料. 由于快淬工艺不同, 它可能形成非晶态或 准晶态结构 ${ }^{[1,2]}$. 关于这种材料的准晶态结构、相变过程及磁性质, 我们已做过系统的研究 ${ }^{[2]}$. 与这一成分相近的非晶态 $\mathrm{ErCo}_{3.44}$ 的磁性质也已由 Jouve 等人进行过研究 ${ }^{[1]}$. 结果表明：(1) 准晶态及非晶态 $\mathrm{Er}_{x} \mathrm{Co}_{100-x}$ 均存在着抵消温度 $T_{\text {comp; }}$ (2) 当 $T>T_{\text {comp }}$ 时, 非晶态及准晶态 $\mathrm{Er}_{x} \mathrm{Co}_{100-x}$ 的磁化强度远高于晶态 $\mathrm{Er}_{x} \mathrm{Co}_{100-x}$ 的磁化强度；(3) 非晶态及准晶态 $\mathrm{Er}_{x} \mathrm{Co}_{100-x}$ 的居 里温度远高于相应晶态的居里温度. Polk 指出, 对于金属间的非晶态结构, 可以用硬球密堆 积模型描述, 在这种结构中含有 $86.2 \%$ 的正四面体 ${ }^{[3]}$. 准晶态 $\mathrm{Er}_{x} \mathrm{Co}_{100-x}$ 的结构为正二十面体, 这种结构可以用两组夹角不同的菱形体来描述 ${ }^{[4]}$, 而这种菱形体又可近似地认为由两个四面 体组成. 因此, 四面体结构成为非晶态和准晶态 $\mathrm{Er}_{x} \mathrm{Co}_{100-x}$ 的结构基元. 为了对这类材料的上 述性质给出解释,我们用 $\mathrm{MS}-\mathrm{X}_{\alpha}$ 方法计算了 $\mathrm{ErCO}_{4}$ 正四面体原子簇的能带结构及电子分布, 计算结果有助于这个问题的解决.

\section{1 物 理 模 型}

正四面体 $\mathrm{ErCo}_{4}$ 原子簇具有 $T_{\mathrm{d}}$ 点群对称. 取 $\mathrm{Er}$ 离子占据正四面体的中心位置, $\mathrm{Co}$ 离子 位于正四面体的顶点. 采用 $\mathrm{MS}-\mathrm{X}_{\alpha}$ 方法对 $\mathrm{ErCO}_{4}$ 原子簇的能带结构和电子态密度进行了计 算.

\section{2 计算方法和过程}

考虑以 $\mathrm{Er}$ 原子核为中心、半径为 $R_{\mathrm{w}}=r_{\mathrm{e}}+2 r_{\mathrm{c}}$ 的 Watson 球, 其中 $r_{\mathrm{e}}$ 和 $r_{\mathrm{c}}$ 分别为 $\mathrm{Er}$ 和 $\mathrm{Co}$ 的原子半径. 在原子球内、原子球之间以及 Watson 球外三个区域内分别求解 $\mathrm{H}-\mathrm{F}-\mathrm{S}$ 方 程

$$
\left[-\frac{1}{2} \nabla^{2}+V^{(i)}(r)\right] \psi_{i}(r)=\varepsilon_{i} \psi_{i}(r),
$$

式中势能 $V$ 的上标 $I=1,2,3$, 分别代表三个不同的区域. 在原子球内 $V^{(1)}(r)$ 是以原子核为中 心的球对称势. 在 Watson 球外 $V^{(3)}(r)$ 也近似地认为是球对称势. 在此二区域内, 方程 (1) 的 解具有径向波函数与球谐函数乘积的形式. 但在原子球间的区域, 由于形状复杂, 势能 $V_{(r)}^{(2)}$ 也

1993-06-09 收稿, 1994-01-04 收修改稿.

*磁学国家重点实验室资助课题. 
具有复杂的形式. 考虑到原子簇的电荷分布主要集中在各原子球内, 球间的电荷密度很小, 所以我们可近似地将 $V^{(2)}(r)$ 作体积平均, 把它作为一个常数势. 已有的计算表明, 这种近似不 会产生较大的误差. 在常数势近似下, 方程 (1) 的解具有多中心形式.

在三个区域分别解方程 (1), 并根据波函数及其一阶导数在边界上连续的性质, 将三个区 域的波函数在边界上分别进行光滑连接, 从而求得原子簇的波函数、能级及电子态密度.

在计算中, 将 $\mathrm{Co}$ 和 $\mathrm{Er}$ 原子的统计平均交换参数 $\alpha$ 分别取为 0.71018 和 $0.69385^{[5]}$; 根据晶 态合金 $\mathrm{ErCo}_{4}$ 的晶格参数并使正四面体 $\mathrm{ErCO}_{4}$ 中的原子刚好相切, 求得 $r_{\mathrm{e}}=1.7766 \AA$, $r_{\mathrm{c}}=1.7537 \mathrm{~A}$; 取原子簇和 Watson 球为电中性, 将 $\mathrm{Co}$ 原子的 $3 d, 4 s$ 电子和 $\mathrm{Er}$ 原子的 $4 f, 6 \mathrm{~s}$ 电 子作为价轨道电子处理. 计算它们的能级及态密度的分布.

\section{3 结果与 讨 论}

对于能级分布的计算结果示于图 1, 图中 $a, e, t$ 分别表示 $1,2,3$ 重简并态, 虚线代表费

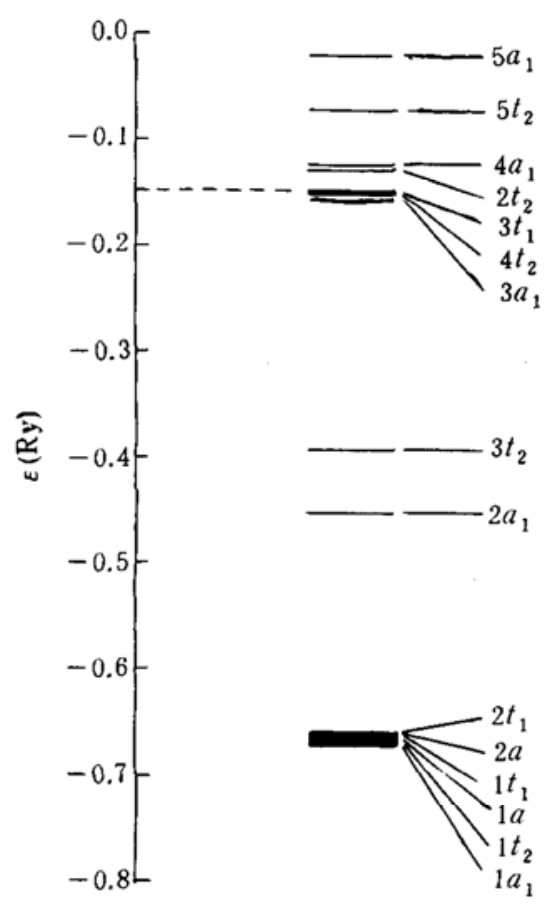

图 $1 \mathrm{ErCO}_{4}$ 原子筷能级

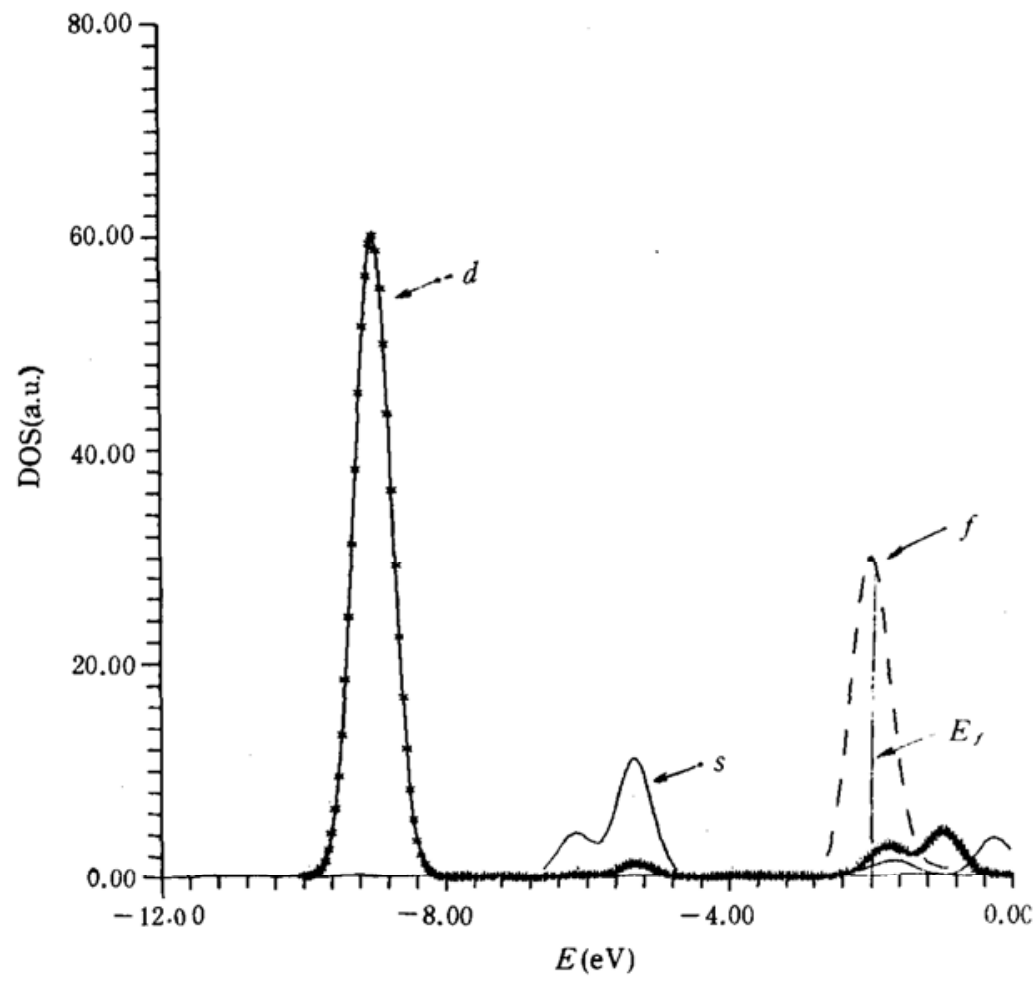

图 $2 \mathrm{ErCo}_{4}$ 原子筷的 $s, d, f$ 能带电子态密度

米面. 对于 $s, d, f$ 能带电子态密度的计算结果示于图 2, 图中的竖直线代表费米面. 根据所 得的计算结果, 可作如下的讨论:

1. 计算所得的某些能级密集在一起, 形成了窄能带. 比较明显的有 3 个能带, 其中居于 能级最深处的 $d$ 能带最为密集, 可以容纳的电子数最多. $s$ 带比较扩展, 可以容纳的电子数最 少. $f$ 带位于费米面附近, 易于被激发.

2. 同金属 $\mathrm{Co}$ 和金属 $\mathrm{Er}$ 的能带结构相比, $\mathrm{ErCO}_{4}$ 原子簇的 $3 d$ 电子和 $4 f$ 电子填充数没有 明显变化, 因而可以推测 $\mathrm{Co}$ 原子和 $\mathrm{Er}$ 原子的磁矩同在金属状态无大的差别. 这一点同下面 
的实验结果是相符合的: 对于准晶态 $\mathrm{ErCO}_{4}$, 实验所得的 $\mathrm{Co}$ 原子磁矩为 $\sigma_{\mathrm{Co}}=1.67 \mu_{\mathrm{B}}$, 分子磁 矩 $\sigma=1.28 \mu_{\mathrm{B}}$; 对于非晶态 $\mathrm{ErCo}_{3.44}$, 实验所得的 $\mathrm{Co}$ 原子磁矩为 $\sigma_{\mathrm{Co}}=1.65 \mu_{\mathrm{B}}$, 分子磁矩 $\sigma=2.13 \mu_{\mathrm{B}}$. 如取 $\mathrm{Er}$ 原子在 $0 \mathrm{~K}$ 时的磁矩为 $7.8 \mu_{\mathrm{B}}^{[1,6]}$, 则准晶态或非晶态 $\mathrm{ErCo}_{4}$ 的磁结构应为散亚铁磁性 结构.

3. 在低温下, $\mathrm{Er}$ 原子的磁矩大于 $\mathrm{Co}$ 原子的磁矩, 因此磁化强度的方向同 $\mathrm{Er}$ 原子的磁矩 方向相一致. 但是, $\mathrm{Er}$ 原子的 $4 f$ 能级位于费米面附近, 易受热激发的影响, 当温度上升时磁矩 下降较快; $\mathrm{Co}$ 原子的 $3 d$ 能带位于能级深处, 不易受热激发的影响, 当温度上升时磁矩下降较 慢. 因此, 在某一温度下, 两种不同方向的磁矩可能变得大小相等, 互相抵消. 此即抵消温度

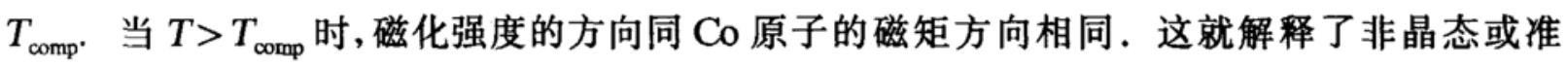
晶态 $\mathrm{Er}_{x} \mathrm{Co}_{100-\mathrm{x}}$ 的磁化强度随温度的变化.

\section{参考文献}

[1] Jouve, H. et al., AIP Conf. Proc., 1976, 29:97; Coey, J. M. D., J. Appl. Phys., 1978, 49: 1646.

[2] Li Hua, Jiang Shouting et al., Acta Mettall. Sinica, 1988, A1: 147.

[3] Polk, D. E., Acta Mettall., 1972, 20: 485.

[ 4] Steinhardt, P. J., Livine, D. et al., Phys. Rev. Lett., 1984, 53: 2477.

[ 5] Schwarz, K., Phys. Rev., 1972, B5: 2466.

[6] Buschow, K. H. J., Fast, J. F., Van der Groot, A. S., Phys. State Solid, 1968, 29: 825. 